\title{
Erosion of a granular bed by an oscillating foil
}

\author{
A. Sauret ${ }^{1}$, C. Morize ${ }^{2}$, P. Gondret ${ }^{2}$ \\ 1. SVI, UMR 125 CNRS/Saint-Gobain, France 2. FAST, CNRS/Paris-Sud University, Orsay, France
}

In various physical situations, a granular bed is resuspended by a flow of air or water. For instance, when a helicopter lands in dusty environments, its blades trigger air recirculation which leads to the resuspension of particles and a limited visibility.

In some cases, the resuspension of particles is sought. Bottom-dwelling fish such as flounders, have indeed found a way to resuspend sand in order to cover themselves and hide. By oscillating their fins, they create a recirculating flow that lifts the particles up and deposits them on top of their backs. Using an oscillating foil placed above a granular bed as a model system, we characterize the fluid motion triggered by vertical displacements of the foil, its influence on the erosion of the granular bed and the erosion pattern.

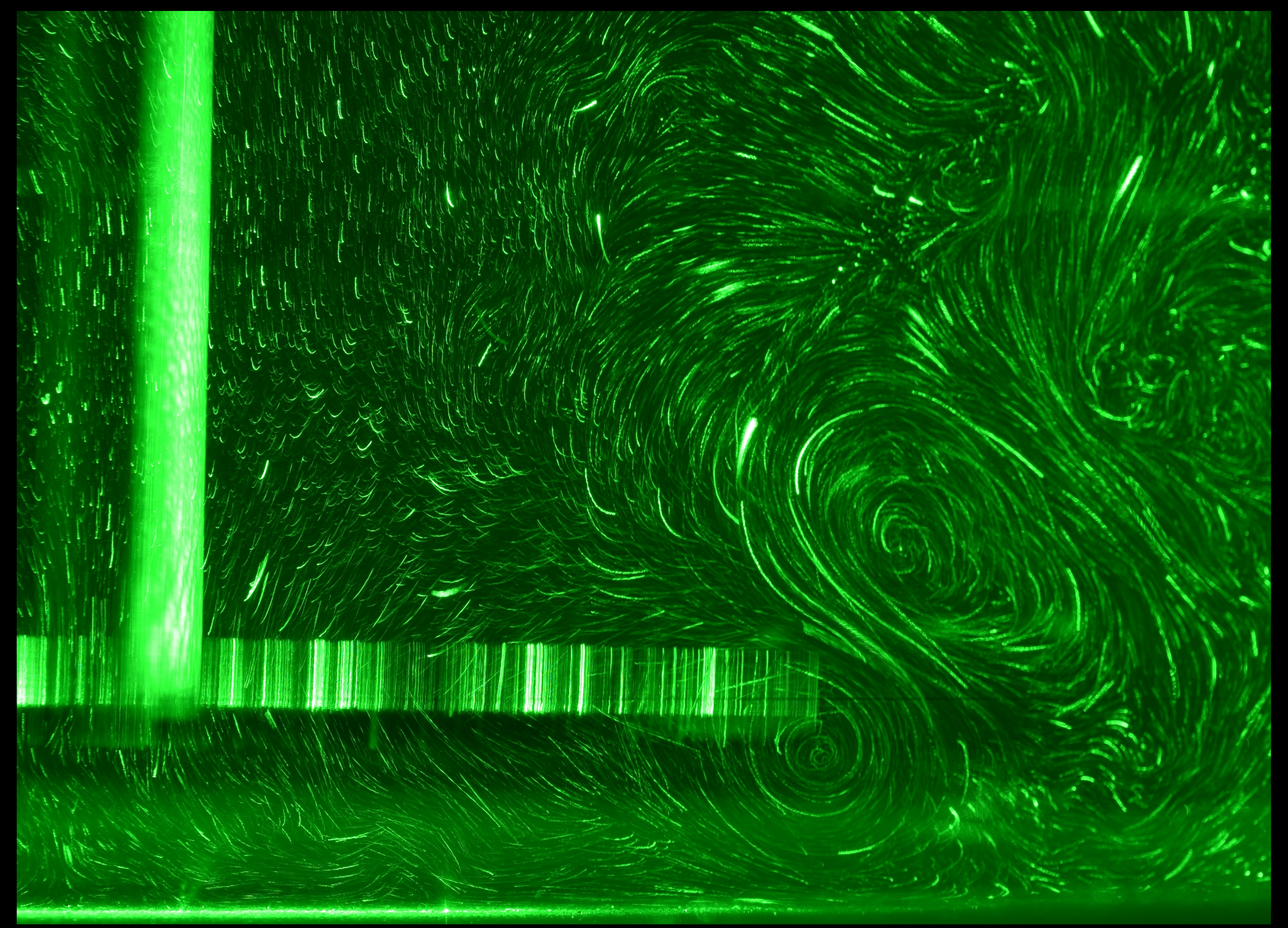

The flow field generated by the oscillating foil leads to various erosion patterns.
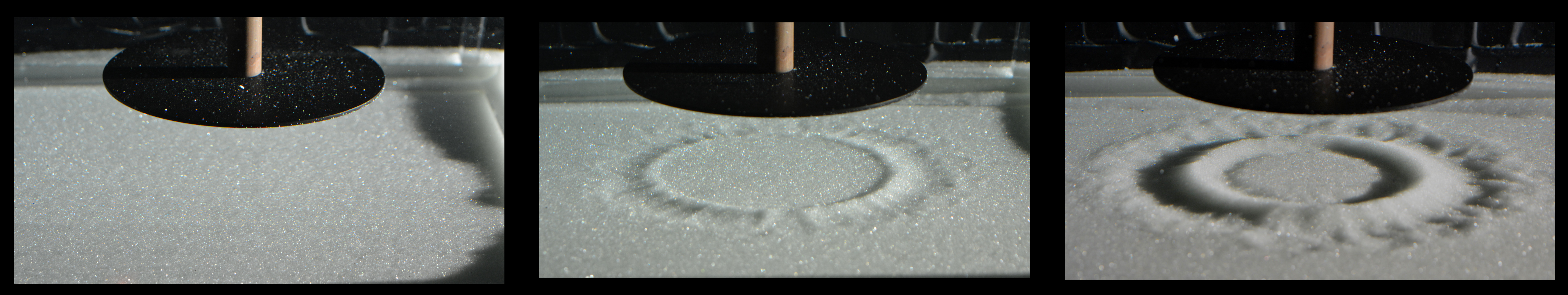